\section{Endocarditis marántica como presentación de cáncer de páncreas. Caso clínico}

\author{
ALEJANDRA SALINAS G. ${ }^{1, a}$, LUIS BUSTAMANTE H. ${ }^{1, a}$, \\ FERNANDO LANAS Z. ${ }^{1,2,3}$, ALVARO SOTO V. ${ }^{4,5}$, \\ MARÍA ANGÉLICA GARCÍA B. ${ }^{1,2}$, CONSTANZA BARTOLOTTI H., ${ }^{1, a}$
}

\section{Non-bacterial thrombotic endocarditis. Report of one case}

Marantic or nonbacterial thrombotic endocarditis is characterized for the presence of vegetations formed by a meshwork of fibrin and other cellular material similar a blood clot, without the presence of microorganisms. It is often related with tumors and chronic inflammatory states. We report a 49 years old female with a history of weight loss and asthenia, presenting with multiple cerebrovascular attacks and fever. Blood cultures were negative and the fever did not subside with antibiotic treatment. Trans esophageal echocardiogram showed a mitral valve vegetation and thickening of the free edge of both leaflets. In search of the etiology of such a case, a primary pancreatic cancer with distant metastases was found. We cannot rule out the differential diagnosis with bacterial endocarditis with negative blood cultures, although the clinical context supports a non-infectious etiology.

(Rev Med Chile 2017; 145: 1353-1358)

Key words: Endocarditis, Non-Infective; Pancreatic Neoplasm; Paraneoplastic Syndromes; Stroke.
'Departamento de Medicina

Interna, Universidad de La

Frontera, Temuco, Chile.

2Departamento de Cardiología,

Hospital Dr. Hernán Henríquez

Aravena, Temuco, Chile.

${ }^{3}$ Capacitación, Investigación y

Gestión para la Salud basada en

Evidencias, Universidad de La

Frontera, Temuco, Chile.

${ }^{4}$ Unidad de Neurología, Hospital

Dr. Hernán Henríquez Aravena,

Temuco, Chile.

${ }^{5}$ Departamento de Especialidades Médicas, Facultad de Medicina,

Universidad de La Frontera.

Temuco, Chile.

${ }^{a}$ Residente de Medicina Interna,

Universidad de La Frontera,

Temuco, Chile.

Recibido el 5 de julio de 2016, aceptado el 6 diciembre de 2016 .

Correspondencia a:

Alejandra Salinas Gallegos

Manuel Montt 115

Departamento de Medicina

Interna, Universidad de La

Frontera.

a.salinasgallegos@gmail.com
L a asociación entre neoplasia maligna y enfermedad tromboembólica venosa fue descrita por Trousseau en el año $1865^{1}$. Más de cien años después, en 1988, Ziegler, describió la endocarditis marántica (EM), también llamada endocarditis trombótica no bacteriana, caracterizada por depósitos de trombos y fibrina sobre válvulas cardíacas. Estudios posteriores identificaron la relación entre EM y estados inflamatorios crónicos, incluyendo neoplasias, septicemia y quemaduras extensas $^{2,3}$.

Presentamos el caso de una mujer de 49 años con endocarditis marántica asociada a una neoplasia pancreática desconocida al ingreso con metástasis a distancia. Se revisan aspectos epidemiológicos y clínicos de esta entidad, cuyo diagnóstico representa un verdadero desafío al médico clínico.

\section{Caso clínico}

Mujer de 49 años con antecedente de obesidad que consultó con historia de 1 día de evolución caracterizada por angustia, insomnio y disnea a lo que se agregó dificultad para emitir lenguaje, parestesias en extremidades, malestar general y fiebre. Dirigidamente se obtuvo el antecedente de astenia y baja de 15 kilos, aproximadamente, en 6 meses.

En su primera evaluación se describió una presión arterial de 129/92 $\mathrm{mmHg}$, temperatura axilar de $37,4^{\circ} \mathrm{C}$, frecuencia respiratoria de 27 por minuto, frecuencia cardiaca regular 98 latidos por minuto, glicemia capilarde $87 \mathrm{mg} / \mathrm{dl}$. Mucosas húmedas, yugulares ingurgitadas a $45^{\circ}$. Examen cardiaco: ritmo regular en 2 tiempos con soplo protomesosistólico de regurgitación en foco mi- 
tral II/IV, sin irradiación. Examen pulmonar con murmullo pulmonar conservado y crépitos desde el tercio medio a las bases, examen abdominal y de extremidades sin alteraciones evidentes. Al examen neurológico se encontraba vigil, inatenta, con afasia de comprensión, sin déficit motor. La tomografía axial computada (TAC) de cerebro sin contraste no mostró lesiones agudas evidentes (Figura 1). El estudio de líquido cefalorraquídeo (LCR) se describió como opalescente incoloro, 24 $\mathrm{mg} / \mathrm{dl}$ de proteínas, glucosa $65 \mathrm{mg} / \mathrm{dl}$, recuento de leucocitos $2 / \mathrm{mm}^{3}$, recuento de hematíes 1.830 / $\mathrm{mm}^{3}$ (punción traumática). El cultivo de LCR fue negativo. Todos exámenes de laboratorio realizados fueron normales.

La paciente ingresó a la Unidad de Medicina Interna con los diagnósticos de infarto de arteria cerebral media izquierda, síndrome febril y sospecha de endocarditis infecciosa. El electrocardiograma se encontraba en ritmo sinusal, sin signos de reacción de cavidades ni alteración de la conducción. La radiografía de tórax mostró signos de congestión pulmonar (Figura 2).

En el hemograma destacó ausencia de leucocitosis y neutrofilia. No obstante, presentó elevación considerable de reactantes de fase aguda y en el examen de orina destacó microhematuria de 3-7/ campo, sin otras alteraciones. Los exámenes de laboratorio están detallados en la Tabla 1.

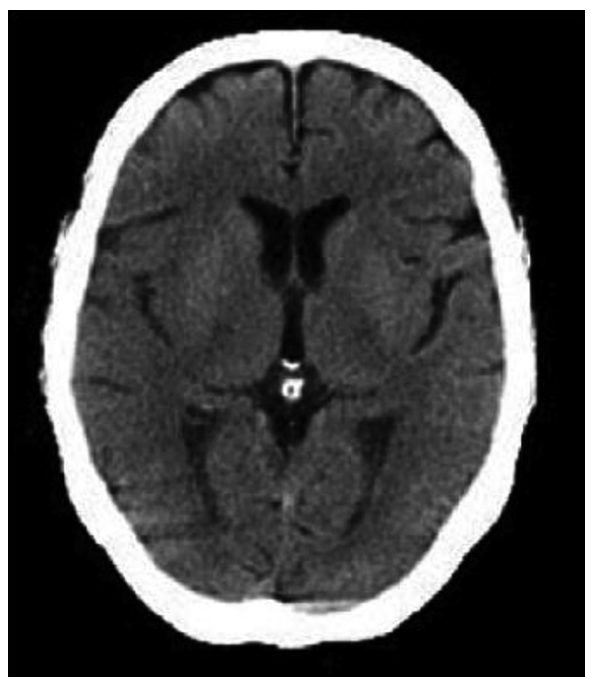

Figura 1. TAC de cerebro sin contraste realizado al ingreso, con $8 \mathrm{~h}$ del inicio de los síntomas. Sin evidencias de lesiones agudas.
Tabla 1. Exámenes de ingreso

\begin{tabular}{|ll|}
\hline Leucocitos & $5,8 \times 109 / \mathrm{L}$ \\
\hline Neutrófilos absoluto & $3,14 \times 10^{9} / \mathrm{L}$ \\
\hline Linfocitos absoluto & $0,55 \times 10^{9} / \mathrm{L}$ \\
\hline Hematocrito & $27,2 \%$ \\
\hline VCM & $88 \mathrm{fL}$ \\
\hline CHCM & $31,9 \%$ \\
\hline Plaquetas & $431.000 / \mathrm{mm}^{3}$ \\
\hline Proteína C reactiva & $180 \mathrm{mg} / \mathrm{L}$ \\
\hline VHS & $53 \mathrm{~mm} / \mathrm{h}$ \\
\hline TSH & $0,33 \mathrm{mUl} / \mathrm{ml}$ \\
\hline Complemento C3 & $224 \mathrm{mg} / \mathrm{dl}$ \\
\hline Complemento C4 & $59,2 \mathrm{mg} / \mathrm{dl}$ \\
\hline Glucosa & $97 \mathrm{mg} / \mathrm{dl}$ \\
\hline Albumina & $3,1 \mathrm{~g} / \mathrm{dl}$ \\
\hline Creatinina & $0,57 \mathrm{mg} / \mathrm{dl}$ \\
\hline Sodio & $140 \mathrm{mEq} / \mathrm{L}$ \\
\hline Potasio & $3,9 \mathrm{mEq} / \mathrm{L}$ \\
\hline Cloro & $103 \mathrm{mEq} / \mathrm{L}$ \\
\hline Ca & $10,1 \mathrm{mEq} / \mathrm{L}$ \\
\hline ASAT & $49 \mathrm{U} / \mathrm{L}$ \\
\hline ALAT & $47 \mathrm{U} / \mathrm{L}$ \\
\hline
\end{tabular}

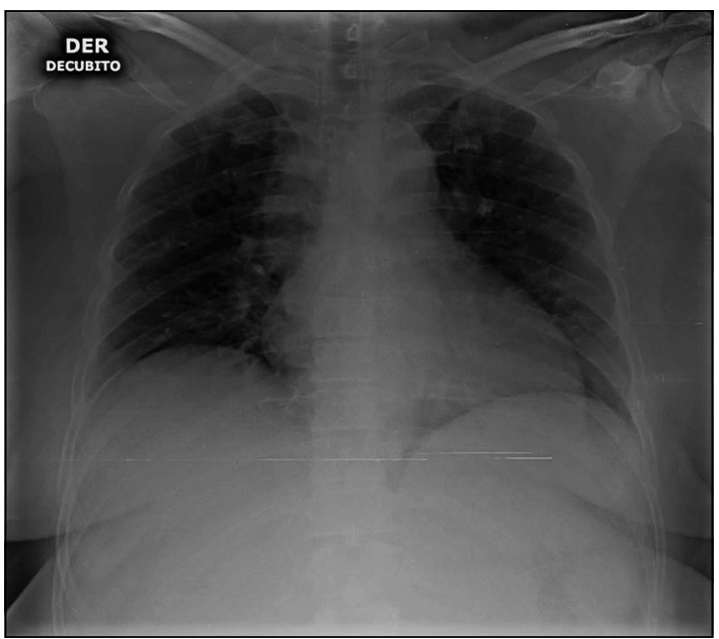

Figura 2. Radiografía de tórax postero-anterior tomada en decúbito que muestra signos de congestión pulmonar, sin focos de condensación. 
Previo al inicio del tratamiento antibiótico se realizó un set de 3 hemocultivos corrientes y 1 hemocultivo en medio para anaeróbios que fueron negativos hasta los 14 días. El urocultivo fue también negativo.

Ante la historia clínica sugerente de endocarditis infecciosa: síndrome febril, déficit neurológico, soplo de insuficiencia mitral, parámetros inflamatorios elevados, anemia normocítica normocrómica y microhematuria, se realizó una ecocardiografía transtorácica que mostró prolapso parcial del velo posterior generando insuficiencia excéntrica, al menos moderada, sin lograr evidenciar con claridad la presencia de vegetaciones. La ecocardiografía transesofágica evidenció una válvula mitral de velos finos con imagen de $5 \mathrm{~mm}$ adherida a la cara auricular de P3 de la válvula mitral, móvil, isoecogénica, compatible con una vegetación. Los bordes libres de ambos velos se encontraron difusamente engrosados. Además, se evidenció una insuficiencia mitral leve.

La paciente evolucionó con deterioro clínico, y persistencia de fiebre hasta $38^{\circ} \mathrm{C}$ a pesar de uso de antibióticos de primera línea para endocarditis en válvula nativa en Chile, penicilina sódica, cloxacilina y gentamicina luego de $72 \mathrm{~h}$ iniciados inmediatamente desde su admisión al servicio de urgencia, luego de la toma de hemocultivos. Continuando con el manejo se tomaron cultivos para microorganismo anaerobios y se escaló en esquema antibiótico a segunda línea ceftiaxona y vancomicina, a pesar de cultivos negativos, no presentar leucocitosis y reactantes de la fase aguda constantemente elevados. Se solicitó una resonancia magnética de cerebro que mostró infartos cerebrales múltiples de la rama posterior de la arteria cerebral media (ACM) izquierda y de la rama anterior de ACM derecha (Figura 3).

En el contexto clínico de endocarditis con hemocultivo negativo, infartos cerebrales múltiples de aspecto cardioembólico y con historia clínica de baja de peso y astenia de 3 meses de evolución se consideró dentro de las hipótesis diagnósticas la posibilidad de una endocarditis marántica como etiología. Se continuó el estudio con marcadores tumorales que resultaron alterados CA 19-9 $>12.000 \mathrm{U} / \mathrm{ml}$, CA $125.234 \mathrm{U} / \mathrm{ml}$, CEA $1.819 \mathrm{ng} /$ $\mathrm{ml}$. Se solicitóTAC de tórax, abdomen y pelvis que mostró una neoplasia primaria pancreática, con infiltración local por vecindad y metástasis hepáticas, esplénicas y renal derecha (Figura 4).

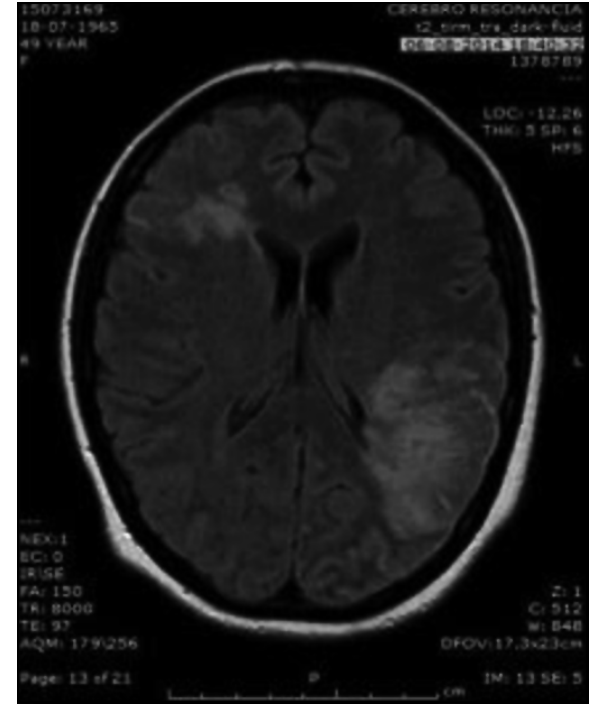

Figura 3. Resonancia nuclear magnética de cerebro. Secuencia $t$ infartos agudos de ambas arterias cerebrales medias, mayor en la izquierda donde hay un pequeño foco de transformación hemorrágico.

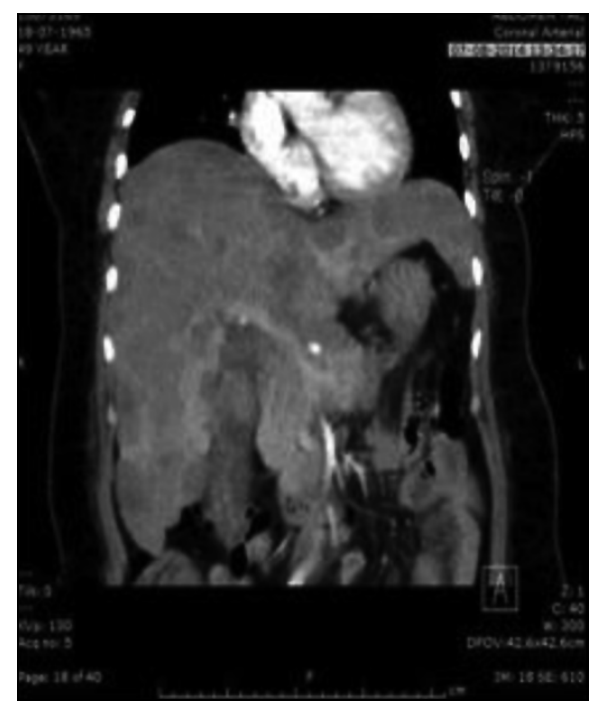

Figura 4. TAC de Tórax, Abdomen y Pelvis. Corte coronal muestra neoplasia primaria pancreática, con infiltración local por vecindad y metástasis hepáticas, esplénicas y renal derecha.

La paciente evolucionó con deterioro clínico progresivo, con compromiso cualitativo y cuantitativo de conciencia. Persistió febril pese a escalada de antibióticos a segunda línea. La paciente falleció a los 10 días desde su ingreso. No se realizó autopsia por voluntad de la familia. 


\section{Discusión}

La Endocarditis marántica, se caracteriza por la presencia de trombos estériles en válvulas cardíacas sin daño previo ${ }^{4}$. El caso presentado demuestra una enfermedad paraneoplásica infrecuente en una paciente con endocarditis trombótica no infecciosa asociado a cáncer de páncreas metastásico como presentación clínica inicial.

Desde las observaciones hechas por el médico francés Armand Trousseau en $1865^{1}$ hasta la fecha, numerosos estudios han confirmado la asociación entre el cáncer y tromboembolismo. La literatura actual indica que el 15\% de los pacientes con cáncer tienen riesgo de sufrir un evento tromboembólico en algún momento del curso de su enfermedad y hasta el $50 \%$ de los pacientes con cáncer tienen evidencia de enfermedad tromboembólica venosa en el examen post mortem ${ }^{2,3}$.

La etiología del estado de hipercoagulabilidad en el cáncer es multifactorial. Los altos niveles del factor de necrosis tumoral y la interleuquina-1, presentes en el cáncer, pueden causar daño en el endotelio de la válvula y formación de trombos ${ }^{3}$. La endocarditis marántica se caracteriza por la alta incidencia de fenómenos embólicos acompañantes, aproximadamente de $42 \%$ (intervalo $14-90 \%$ ) los más frecuentes son los cerebrales ${ }^{4}$; su incidencia varía entre el 0,3 y el 9,3\% en series necrópsicas $\mathrm{y}$ afecta generalmente a pacientes entre la cuarta y la octava década de la vida, aunque puede afectar a personas de cualquier edad 5 .

El mecanismo fisiopatológico de la lesión sigue siendo no precisado, sin embargo, el factor más importante en la formación de las vegetaciones es un estado de hipercoagulabilidad asociado generalmente a una neoplasia maligna como síndrome paraneoplásico y el tratamiento de éstas ${ }^{5}$. Los tipos histológicos más frecuentes son adenocarcinomas originarios del páncreas, estómago, intestino grueso, vesícula biliar, conductos biliares, ovario y pulmón. Siendo especialmente alto el riesgo en cáncer de páncreas en comparación con otros tipos de adenocarcinomas $(10,34 \%$ vs $1,55 \%$ respectivamente) ${ }^{6}$.

La incidencia exacta de la endocarditis marántica es desconocida. Un estudio de serie de autopsias reveló 10 casos en 1.640 adultos, mostrando una prevalencia de $1,25 \%$ en los pacientes con cáncer y $0,2 \%$ en la población general ${ }^{6}$. La EM afecta en su mayoría los pacientes entre la $4^{\mathrm{a}}$ y $8^{a}$ década de vida ${ }^{5}$. Pareciera que hay un ligero predominio por el sexo femenino ${ }^{7}$, aunque otros estudios no informaron predilección de género ${ }^{5}$. Las válvulas más afectadas en orden descendente son: válvula aórtica, válvula mitral y afectación simultánea de la válvula aórtica y mitral ${ }^{5}$.

En cuanto a la presentación clínica los principales síntomas derivan de la enfermedad tromboembólica sistémica. Los sitios más frecuentemente afectados incluyen el bazo, los riñones y las extremidades. Sin embargo, el caso más grave para el paciente es la embolización al sistema nervioso central ${ }^{6}$ como fue el caso de nuestra paciente que exhibía infartos cerebrales múltiples. La diferencia entre endocarditis trombótica e infecciosa puede convertirse en un verdadero dilema para el médico clínico, principalmente porque ninguna característica es patognomónica. Los soplos cardíacos ocurren con poca frecuencia. Es por esto, que la aparición de un nuevo soplo en un paciente con tumor maligno debe dar lugar a sospecha de endocarditis marántica ${ }^{5}$. En nuestro caso la paciente presentaba un soplo proto-mesosistólico de regurgitación en foco mitral II/IV, sin irradiación, pero no sabemos si lo presentaba previamente.

El diagnóstico se realiza a través de la visualización de vegetaciones endocárdicas asociada a la ausencia de microorganismos en la sangre. Al igual que en la endocarditis infecciosa (EI) la ecocardiografía transesofágica (ETE) sigue siendo el método de elección en cuanto al estudio de imágenes ${ }^{8}$. Sin embargo, es importante señalar que las lesiones valvulares de la endocarditis marántica resultan imagenológicamente indistinguibles de las observadas en la EI, por lo que es necesario considerar como diagnóstico diferencial esta entidad ${ }^{11}$. La ETE es el método más sensible para el diagnóstico de vegetaciones. La ecocardiografía transtorácica resulta una técnica útil para su evaluación, pero debido a su menor sensibilidad, las vegetaciones de pequeño tamaño pueden pasar desapercibidas. Por ello, en caso de elevada sospecha clínica con ETT no concluyente, está indicada la realización de una ecocardiografía transesofágica9 ${ }^{9}$. En nuestro caso se realizó en primer lugar una ETT que evidenció un prolapso parcial del velo posterior que generó una insuficiencia excéntrica moderada, a continuación se realizó un ETE que reveló una imagen sugerente de vegetación con el borde de ambos velos engrosado, a pesar de que las ecografías no mostraban signos de insuficiencia 
cardiaca la paciente tenía signos de congestión probablemente debido al sindrome de inflamación sistémica. El valor potencial de la ETE en el diagnóstico de endocarditis marántica se puso de manifiesto en una serie de 51 pacientes con cáncer que presentaron eventos cerebrovasculares, casi la mitad de los pacientes tenían una fuente cardiaca definida para la embolia. Se detectaron vegetaciones no bacterianas en nueve pacientes (18\%). La ETT se realizó en siete de estos pacientes, y fue negativa en cuatro ${ }^{10}$. No se han realizado estudios ecocardiográficos que evalúen de forma prospectiva a pacientes con cáncer que puedan desarrollar una endocarditis marántica.

El tratamiento en estos pacientes se basa en una terapia dirigida a la neoplasia maligna subyacente y anticoagulación sistémica. La anticoagulación más eficaz parece ser la heparina no fraccionada endovenosa en infusión continua, que ha demostrado ser eficaz en la reducción de la incidencia de episodios recurrentes de tromboembolia. En contraste con la heparina, los antagonistas de la vitamina $\mathrm{K}$ tales como warfarina no se deben utilizar en pacientes con endocarditis marántica con neoplasia asociada, ya que son comunes los eventos tromboembólicos mientras se utiliza ${ }^{5,12}$. No obstante, no existen datos concluyentes que aconsejen un tratamiento definitivo. En los pacientes con enfermedad neoplásica diseminada, la anticoagulación se debe considerar si el tumor es sensible a la quimioterapia y/o radioterapia, o si hay alguna otra condición tratable subyacente. $\mathrm{Si}$ no hay esperanza de regresión del tumor, se sugiere no administrar anticoagulantes ${ }^{12,13}$.

Por otro lado, aunque la mayoría de los pacientes no requiere cirugía cardiaca por la endocarditis, en los que desarrollan una insuficiencia valvular grave puede estar indicada la cirugía de recambio valvular si la situación clínica del paciente, el pronóstico de la enfermedad de base y la relación beneficio-riesgo son favorables ${ }^{5}$.

En cuanto al pronóstico, éste varía en relación a la efectividad del tratamiento de la neoplasia primaria. En estudios en cáncer de ovario, una intervención quirúrgica positiva puede influir en gran medida, aunque esto depende de la condición general del paciente cuando se produce la endocarditis marántica ${ }^{14}$. En la mayoría de los casos, la muerte se produjo a consecuencia de la principal enfermedad asociada, 32\% causa neoplásica, en cambio sólo $9,1 \%$ de los casos la causa de la muerte está directamente relacionada con la presencia de endocarditis marántica. Dentro de las complicaciones responsables de la muerte se puede nombrar la herniación cerebral tras embolia en el sistema nervioso central y tromboembolia pulmonar ${ }^{15}$. No obstante no existe mucha información acerca del pronóstico en estos pacientes ya que la mayoría de los casos reportados son hallazgos en la necropsia, en otros casos puede sub diagnosticarse y al tratarse la patología de base y se resuelven las complicaciones secundarias.

El caso clínico presentado aunque sin comprobación anatomopatológica es compatible con endocarditis marántica. La negatividad persistente de los hemocultivos, la ausencia de mejoría clínica con tratamiento antibiótico y la presencia de cáncer de páncreas que es la neoplasia con mayor asociación a esta patología, sugieren evidencias fuertes para pensar como primera opción en este diagnóstico. La presencia de fiebre puede deberse a manifestaciones secundarias a la neoplasia e infiltración maligna. El diagnóstico de EM es de exclusión, por lo que es importante en nuestro medio, dado el bajo rendimiento de los hemocultivos, enfatizar en optimizar sus resultados, tomando más muestras, 3 hemocultivos más, a las horas después de las muestras iniciales o al día siguiente, si la condición clínica del paciente lo permite para no iniciar antibióticos de inmediato, y ponerse en contacto con el laboratorio para realizar técnicas especiales para el estudio de gérmenes fastidiosos. Es necesario el estudio serológico de algunas causas infecciosas si se encuentra disponible. Es importante tener en cuenta esta presentación clínica atípica y realizar un diagnóstico diferencial oportuno, lo que es fundamental en estos casos probablemente no para buscar la resolución de la enfermedad, sino para realizar una labor más compleja aún, como han referido los médicos franceses Bérard y Gubler en el siglo XIX, "Curar pocas veces, aliviar a menudo, consolar siempre".

\section{Referencias}

1. Trousseau A. Clinique Médicale de l'Hôtel-Dieu de Paris. Paris: Ballière; 1865. Phlegmasia alba dolens; p. 654-712.

2. Deitcher SR. Cancer and thrombosis: Mechanisms and treatment. J Thromb Thrombolysis 2003; 16: 21-31. 
3. Dvorak HF. Abnormalities of hemostasis in malignant disease. In: Colman RW, Hirsh J, Marder VJ, et al., editors. Hemostasis and Thrombosis. Philadelphia: J. B. Lippincott; 1994. p. 1238-54.

4. Silver MD, Gotlieb AI, Schoen FJ. Cardiovascular Pathology, Churchill Livingstone, New York, 2001, pp. 447-9.

5. el-Shami K, Griffiths E, Streiff M. Nonbacterial thrombotic endocarditis in cancer patients: pathogenesis, diagnosis, and treatment. Oncologist 2007; 12: 518-23.

6. González-Quintela A, Candela MJ, Vidal C, Román J, Aramburo P. Non-bacterial thrombotic endocarditis in cancer patients. Acta Cardiol 1991; 46: 1-9.

7. Steiner I. Nonbacterial thrombotic versus infective endocarditis: a necropsy study of 320 cases. Cardiovasc Pathol 1995; 4: 207-9.

8. Lee RJ, Bartzokis T, Yeoh TK, Grogin HR, Choi D, Schnittger I. Enhanced detection of intracardiac sources of cerebral emboli by transesophageal echocardiography, Stroke 1991; 22: 734-9.

9. Joffe II, Jacobs LE, Owen AN, Ioli A, Kotler MN. Noninfectivevalvular masses: review of the literature with emphasis on imaging techniques and management. Am Heart J 1996.

10. Lee RJ, Bartzokis T, Yeoh TK, Grogin HR, Choi D, Sch- nittger I. Enhanced detection of intracardiac sources of cerebral emboli by transesophageal echocardiography. Stroke 1991; 22: 734-9.

11. Reisner SA, Brenner B, Haim N, Edoute Y, Markiewick W. Echocardiography in nonbacterial thrombotic endocarditis: from autopsy to clinical entity. Am Soc Echocardiogr 2000; 13: 876-81.

12. Rogers LR, Cho ES, Kempin S, Posner JB. Cerebral infarction from non-bacterial thrombotic endocarditis: Clinical and pathological study including the effects of anticoagulation. Am J Med 1987; 83: 746-56.

13. Salem DN, Stein PD, Al-Ahmad A, Bussey HI, Horstkotte $\mathrm{D}$, Miller $\mathrm{N}$, et al. Antithrombotic therapy in valvular heart disease-native and prosthetic: The Seventh ACCP Conference on Antithrombotic and Thrombolytic Therapy. Chest 2004; 126 (3 suppl): 457S-82S.

14. Tanaka H, Ito M, Yoshida K, Asakura T, Taniguchi H. Nonbacterial thrombotic endocarditis complicated with stage Ia ovarian cancer. Int J Clin Oncol 2009; 14 (4): 369-71.

15. Llenas-García J, Guerra-Vales JM, Montes-Moreno S, López-Ríos F, Castelbón-Fernández FJ, Chimeno-García J. Endocarditis trombótica no bacteriana: estudio clínico-patológico de una serie necrópsica. Rev Esp Cardiol 2007; 60 (5): 493-500. 